\title{
Produção compartilhada de conhecimentos em saúde mental: o Comitê de Acompanhamento de Pesquisa
}

\section{Shared knowledge production in mental health: the Research Monitoring Committee}

\author{
Barbara Coelho Vaz \\ (D) https://orcid.org/0000-0001-9305-9586 \\ E-mail: barbaracvazœgmail.com \\ (iD) Jorge Lyrab \\ https://orcid.org/0000-0002-5381-2059 \\ E-mail: jorglyraœgmail.com \\ Antonio José Costa Cardosoc \\ (D) https://orcid.org/0000-0002-6085-3502 \\ E-mail: antoniojccardoso@gmail.com

\section{Alyne Alvarez Silva ${ }^{d}$ \\ (i) https://orcid.org/0000-0003-1022-326X \\ E-mail: alvarezalyneळgmail.com}

\section{Maristela de Melo Moraes ${ }^{d}$} \\ (iD) https://orcid.org/0000-0002-3622-6824 \\ E-mail: maristelammoraesळgmail.com \\ aFundação Oswaldo Cruz. Núcleo de Saúde Mental, Álcool e \\ Outras Drogas. Brasília, DF, Brasil. \\ bUniversidade Federal de Pernambuco. Centro de Filosofia e \\ Ciências Humanas. Departamento de Psicologia. Recife, PE, \\ Brasil. \\ 'Universidade Federal do Sul da Bahia. Instituto de Humanidades, \\ Artes e Ciências. Itabuna, BA, Brasil. \\ 'Universidade Federal de Campina Grande. Unidade Acadêmica \\ de Psicologia. Campina Grande, PB, Brasil.
}

\section{Correspondência}

Fiocruz Brasília

Campus Universitário Darcy Ribeiro.

Avenida L3 Norte, S/N. Gleba A Bloco Administrativo - Primeiro Andar

Brasília, DF. CEP 70904-130

\section{Resumo}

Esse artigo objetiva refletir sobre o funcionamento do Comitê de Acompanhamento de Pesquisa (CAP) adotado no estudo nacional que avaliou os efeitos do Programa de Volta para Casa (PVC) na vida dos beneficiários em onze municípios brasileiros. A pesquisa foi aprovada por Comitê de Ética em Pesquisa e desenvolvida entre 2015 e 2018. O CAP pode ser entendido como: dispositivo de interlocução, por circular opiniões, dúvidas e críticas dos atores sociais implicados no processo de pesquisa; dispositivo de qualificação da pesquisa, na medida em que definições de procedimentos e análise dos achados passam por processos de negociação entre diferentes atores; e dispositivo gerencial, por permitir recomendações para os serviços envolvidos. Em todos os municípios participantes a instalação do CAP foi pactuada com os gestores de saúde mental. Realizaram-se em média três reuniões por município, com duração mínima de uma hora e 15 participantes por encontro (pesquisadores, gestores, trabalhadores da Rede de Atenção Psicossocial (Raps), cuidadores das residências terapêuticas, beneficiários e familiares, entre outros atores), totalizando 30 reuniões. As informações foram organizadas em três eixos: configurações do CAP, conteúdos discutidos e processos da pesquisa. As pautas mais recorrentes relativas ao PVC foram seus efeitos na vida cotidiana dos beneficiários, seu uso como ferramenta de desenvolvimento de habilidades múltiplas, e dificuldades tanto de implementação do Programa quanto de administração do dinheiro pelos beneficiários. O CAP fomentou protagonismo, fortaleceu a autonomia dos beneficiários, possibilitou a problematização das práticas dos 
profissionais e gestores da Raps e contribuiu para a qualificação do PVC.

Palavras-chave: Pesquisa Participativa Baseada na Comunidade; Ferramenta Metodológica; Saúde Mental.

\section{Abstract}

This article intends to reflect on the functioning of the Research Monitoring Committee (CAP) adopted in the national study that evaluated the effects of the De Volta para Casa Program (PVC - Back Home Program) in the lives of the beneficiaries in eleven Brazilian municipalities. The research was approved by Research Ethics Committee and developed between 2015 and 2018. The CAP can be understood as: a device of interlocution by circulating opinions, questions and criticism of the social actors involved in the research process; a device of research qualification, considering that procedures' definitions and findings' analysis undergo negotiation processes between different actors; and a managerial device, because it allows recommendations to the services involved. In all the participating municipalities, the CAP establishment was agreed upon by the mental health managers. On average, three meetings per municipality were held, lasting at least one hour and gathering 15 participants (researchers, managers, workers of the Psychosocial Care Network (Raps), caregivers of therapeutic residences, beneficiaries and family members, among other actors), in a total of thirty meetings. The information was organized into three axes: CAP configurations, discussed content, and research processes. The most recurrent issues relating to PVC were its effect on the daily life of the beneficiaries, its use as a tool for the development of multiple skills, and difficulties of both the implementation of the program and the money administration by beneficiaries. The CAP promoted protagonism, strengthened beneficiaries' autonomy, enabled the problematization of the practices of the professionals and managers of the Raps, and contributed to the qualification of the PVC. Keywords: Community-Based Participatory Research; Methodological Tool; Mental Health.

\section{Considerações iniciais}

Pesquisas avaliativas de políticas públicas podem ser desenvolvidas utilizando diferentes metodologias, mas, por dever ético-político, o método deve ser coerente com os princípios que orientam a política sob escrutínio. Por essa razão, no caso do Sistema Único de Saúde (SUS), tem se valorizado cada vez mais as pesquisas participativas, visando garantir a inserção do maior número possível de participantes no processo investigativo, o que obriga a reconsiderar o lugar do(a) pesquisador(a) e do sujeito da pesquisa na produção do conhecimento (Diaz, 2009; Stake, 2003).

A experiência que apresentaremos neste texto se sustenta na ideia de que uma metodologia de pesquisa pode ser compreendida como processo negociado e argumentado de escolhas (Spink; Menegon, 1999), sendo a narrativa produzida, no final das contas, uma construção retórica, e não uma história que resgata a veracidade dos fatos com fidedignidade descritiva. Essa perspectiva crítica da ciência orienta o recorte do objeto, a definição de caminhos de leitura e a construção da pergunta norteadora e do problema de investigação (Lyra; Medrado, 2010).

Nas pesquisas qualitativas em saúde, por exemplo, são empregadas diversas estratégias de acesso às informações e práticas interpretativas (Denzin; Lincoln, 20o6) que geram um conjunto complexo de etapas, cuja trajetória, muitas vezes, somente encontra linearidade na elaboração do texto final (Lyra; Medrado, 2010). Pensar a pesquisa como "jogo semicontrolado", que leva em consideração não só o lugar e os interesses dos(as) pesquisadores(as), mas também dos sujeitos da pesquisa, abre a possibilidade de repensar a produção do conhecimento sem abrir mão do rigor metodológico, como um exercício coletivo, negociado, de escolhas (Denzin; Lincoln, 2006).

A principal característica do que chamamos de um jogo semicontrolado está no seguinte: há aspectos e momentos do jogo em que, apesar dos outros jogadores, pode-se calcular resultados com alta margem de segurança ou com probabilidades. [...] O sistema torna-se mais previsível. Mas há 
outros aspectos e momentos do jogo em que só se pode fazer apostas condicionadas à ocorrência de determinadas circunstâncias e decidir apenas na base de preferência quanto a alguma aposta, pois o cálculo de resultados é impossível. [...] Nesse último caso, o futuro é nebuloso, difuso e indeterminável. Não se pode calcular o risco de uma jogada ou de uma decisão. A incerteza é inexorável. 0 que o plano [projeto] anuncia é uma aposta débil (MATUS, 2006, p. 118).

No campo da saúde mental, coerentemente com os princípios da reforma psiquiátrica brasileira de horizontalidade, dialogicidade, participação e inclusão, foram desenvolvidas inovações metodológicas, tendo como referência o que Spink (200o) propõe como "princípios de uma ética dialógica" em lugar de uma "ética prescritiva”. Um exemplo disso é o Comitê de Acompanhamento da Pesquisa (CAP), de que tratará este artigo.

O CAP pretende fortalecer o protagonismo dos(as) usuários(as) e ampliar a participação de interlocutores(as) não tradicionais - usuários(as), familiares, gestores(as) e outros(as) - na construção coletiva de conhecimento dialógico e, dessa forma, superar a dicotomia sujeito-objeto e a pretensa objetividade científica que lhe é correlata.

Essa pesquisa lançou mão da triangulação metodológica, bem como da triangulação de pesquisadores(as), para avaliar os efeitos do Programa de Volta para Casa (PVC), discutindo a importância do CAP como estratégia democrática indutora da participação dos(as) diversos(as) participantes envolvidos(as) com o objeto/tema pesquisado. Propôs-se com esta metodologia estabelecer relações horizontais e dialógicas que permitam a eles opinar sobre "como, quando e quem" pode participar, bem como contribuir com reflexões e análises acerca dos acontecimentos ocorridos no campo.

A triangulação metodológica, segundo Minayo, Assis e Souza (2005), expressa uma dinâmica de investigação e de trabalho que integra análises de estruturas, processos e resultados. Na opinião de Flick (1998), trata-se de estratégia que pretende conferir maior complexidade e profundidade à pesquisa, produzindo mais elementos para a interpretação. Saupe e Wendhausen (2006) destacam, entretanto, que essa triangulação pode ser referida ao uso de procedimentos semelhantes de coleta e análise de dados em múltiplas fontes que podem variar em relação ao tempo e ao espaço (triangulação de dados); ao estudo de um objeto ou problema de pesquisa gerado a partir de diferentes campos disciplinares (triangulação teórica); e ao emprego de estratégias complementares de coleta e análise de dados (triangulação metodológica).

Menos conhecida ou usual é a triangulação de pesquisadores(as). Caracterizada pelo envolvimento de indivíduos diferentes, seja na realização da coleta de dados por diferentes meios, gerando um conjunto diverso de informações, seja no processo de análise sobre as mesmas informações, a triangulação de pesquisadores(as) é empregada em pesquisas quantitativas que visam a "avaliação por juízes" para garantir maior validade a instrumentos de análise ou avaliação, e também em pesquisas qualitativas que pretendem assegurar a construção de análise mais qualificada, densa e plural, garantindo o rigor metodológico na medida em que a definição de procedimentos e categorias passa por processos de negociação entre diferentes atores (Koizumi, 1992; Lyra; Medrado, 2010; Saupe; Wendhausen, 2006).

A partir da descrição da proposta inicial de configuração e finalidade do CAP para esta pesquisa, pretende-se refletir sobre sua contribuição potencial em pesquisas avaliativas participativas, além de analisar as diversas maneiras de sua realização. Nesse sentido, segue-se uma análise do dispositivo, considerando diferentes modelagens nos campos da pesquisa em tela.

\section{Concepção}

Zuñiga e Laperrière (2006) compreendem a pesquisa avaliativa participativa sob a premissa de que as opções metodológicas permanecem nas sombras do implícito e ocultam as opções sociopolíticas dos(as) pesquisadores(as), por sua vez afetados(as) pelas mudanças propostas. Duas experiências serviram de base para suas reflexões: as atividades de prevenção de DST/aids realizadas na região amazônica e a construção coletiva de uma ferramenta de avaliação em Quebec. 
A partir dessas duas experiências, dois problemas principais surgiram: participação e sustentabilidade real; e prestação de contas à fonte de financiamento. Especificamente sobre a experiência brasileira, segundo os autores, outras duas questões surgiram quando analisadas: a imprevisibilidade e a verticalidade. Tendo em vista as condições reais de vida dos(as) participantes (locais de moradia, segurança, transporte, modos de vida e subsistência), essa participação se dá sempre diante de contradições, imprevistos e limites com os quais a pesquisa precisa lidar, e "com os quais o projeto de pesquisa não pode negociar como o faz com os pares 'visíveis e reconhecidos' do sistema oficial de saúde” (Zuñiga; Laperrière, 2006, p. 120-121).

$\mathrm{Na}$ experiência dos referidos autores, as questões citadas só puderam ser administradas quando se criou um grupo de pessoas das comunidades objeto da pesquisa para compartilhar as decisões relativas aos procedimentos da investigação. 0 grupo, cuja finalidade seria lidar com os principais problemas daquelas experiências, ficou conhecido como CAP. Ao promover o protagonismo dos(as) usuários(as), ampliou a potência dos resultados e favoreceu processos permanentes de discussão, superando limitações tradicionais do processo de pesquisa. 0 dispositivo colaborou, além disso, para o acesso e incorporação do conhecimento produzido pela comunidade.

O CAP pode ser entendido, assim, como um mecanismo de interlocução entre pesquisadores(as), trabalhadores(as) e gestores(as), bem como usuários(as) dos serviços e seus(suas) familiares, militantes de movimentos sociais e outros(as) participantes da comunidade implicados(as) no campo em questão. Nesse sentido, o CAP formaliza um canal de comunicação por meio do qual circulam opiniões, dúvidas e críticas à pesquisa em andamento, constituindo-se em um espaço de troca de informações com os atores envolvidos e de problematização das questões que emergem no decorrer da pesquisa.

No Brasil, o CAP (ou dispositivo análogo) já foi utilizado em pesquisas participativas e avaliativas no contexto da saúde mental. Onocko-Campos et al. (2008), em pesquisa avaliativa, denominaram tal dispositivo de "comitê científico". Passos et al. (2013) adotaram o Comitê Cidadão como estratégia de cogestão em uma pesquisa participativa no campo da saúde mental. Ambas dizem ser possível constatar como a ferramenta contribuiu para a interlocução entre o grupo dos(as) pesquisadores(as) e a equipe operacional e técnica do município. Para estes, as reflexões sobre a prática e o trabalho em rede foram fundamentais para a superação de alguns entraves da gestão da pesquisa.

Em outra importante pesquisa sobre o morar e o habitar de pessoas egressas de longas internações psiquiátricas (Furtado; Nakamura, 2014), estabeleceu-se o CAP para servir de canal de comunicação e circulação das opiniões, dúvidas e críticas, enfim, um espaço de troca de informações sobre o andamento da pesquisa com os atores envolvidos. Considerou-se o espaço - no qual todos têm a oportunidade de se expressar - oportuno para discutir questões que permearam o objeto estudado, o que teria enriquecido a compreensão dos(as) pesquisadores(as).

Com essas reflexões em vista, adotou-se o CAP nesta pesquisa de avaliação do PVC. O ideal teria sido conceber o projeto da pesquisa já com a colaboração do grupo, mas a logística impediu por conta da negociação, tempo do financiamento, entre outros fatores. Portanto, a constituição dos CAP se deu no início da execução da investigação. No entanto, a experiência vivida nos deu subsídio para propor, como objetivo deste artigo, a avaliação desta ferramenta.

\section{Procedimentos}

A pesquisa em tela foi desenvolvida entre 2015 e 2018, tendo sido aprovada pelo Comitê de Ética em Pesquisa da Fundação Oswaldo Cruz - Brasília (Fiocruz/DF), com parecer n ${ }^{0} 1.699 .082$.

Para a constituição dos Comitês de Acompanhamento da Pesquisa nos 11 municípios brasileiros onde foi realizada, pactuou-se com os(as) gestores(as) municipais de saúde mental a sua implantação. Além de apresentar a pesquisa e a necessidade de fazê-la tramitar nos comitês de ética locais - exceto nos municípios que não demandaram essa aprovação -, pactuou-se que apenas com a autorização dos(as) gestores(as) se daria início à pesquisa de campo. Esclareceu-se também que os 
objetivos do CAP estão relacionados à qualificação da pesquisa e à garantia da participação plural dos atores envolvidos nesse processo de produção de conhecimento.

Foi definida uma composição mínima (ideal, tentativa) de atores para o CAP, visando garantir ampla representatividade e densa discussão: gestor de saúde mental do município, gestores dos serviços envolvidos, beneficiários, técnicos responsáveis pelo programa e trabalhadores dos serviços, podendose agregar representantes do Ministério Público e movimentos sociais, entre outros atores-chave.

Definiu-se, como meta, a necessidade de realizar, ao menos, três reuniões de cada CAP, sendo a primeira para apresentação do projeto de pesquisa, e a segunda e terceira para discussão dos achados e encaminhamentos, bem como outras reuniões que poderiam ser realizadas em caráter extraordinário, caso houvesse demanda.

Depois de pactuada a realização da pesquisa, foram feitos os convites para a primeira reunião em cada município, cujo objetivo foi apresentar a pesquisa e a ferramenta CAP para os(as) participantes, fazer acordos para o seu funcionamento e solicitar informações para a localização dos(as) beneficiários(as) do PVC selecionados(as) para a pesquisa. Considerando a necessária delicadeza em pesquisas com usuários(as) de serviços da saúde mental, uma preocupação permanente nessa primeira reunião era combinar a estratégia de aproximação dos(as) pesquisadores(as) ao campo, como adentrar os serviços ou residências, espaços de convívio e cotidianos sem interferir muito na rotina, caso fosse necessário contatar os(as) beneficiários(as) nesses locais.

As demais reuniões foram dedicadas ao andamento da pesquisa, inclusive em alguns CAP, e à leitura e discussão de trechos das narrativas construídas pelos(as) pesquisadores(as) contando as trajetórias de vida dos(as) beneficiários(as) no que diz respeito aos diálogos possíveis da díade dinheiro-autonomia, que protagonizou uma das principais perguntas da investigação: "o que o dinheiro faz poder?"

Para a análise dos dados, em cada campo as reuniões realizadas foram transcritas, agregando-se informações-chave produzidas pelos pesquisadores de campo. Essas informações foram dispostas em matriz de análise organizada em três grandes eixos - configurações do CAP (participantes, número de reuniões, entre outras), conteúdos discutidos e processo da pesquisa em curso -, o que permitiu fazer emergir as "categorias temáticas".

Havia também a expectativa de que o CAP e o processo de pesquisa participativa permitiriam, ao final, sugerir recomendações e/ou encaminhamentos para a Rede de Atenção Psicossocial (Raps) e o programa no âmbito municipal, mas isso nem sempre foi possível.

\section{Resultado e discussão}

De acordo com a experiência de Furtado e Nakamura (2014), o CAP é um espaço de circulação de opiniões, dúvidas e críticas dos atores envolvidos acerca do andamento da pesquisa, sendo oportuno para discutir questões que permeiam o objeto estudado num espaço no qual todos podem se expressar perante o grupo. Sendo assim, nesta investigação o CAP se constitui não só como um dispositivo operacional de pesquisa, mas também como um dispositivo gerencial e de participação (Passos et al., 2013).

Na categoria configuração do CAP, conseguiu-se realizar um total de 30 reuniões de CAP durante a pesquisa nas 11 cidades selecionadas. Em média, foram realizadas três reuniões em cada cidade, com duração mínima de uma hora, todas registradas em gravador digital e degravadas na íntegra pela equipe de pesquisa.

Em todos os municípios, a pauta mais recorrente foi o que acontecia com o PVC localmente e de que modo esse benefício impactava a vida de seus(suas) beneficiários(as) quanto ao aumento da contratualidade e autonomia. Levantaram-se, no cenário municipal, as dificuldades de implementação cotidianas do Programa, a importância do benefício na vida das pessoas e as dificuldades de algumas delas em administrar o dinheiro e demandar apoio. Nessas descrições, o CAP atuou próximo de uma outra experiência de pesquisa participativa que focou no processo de autonomia foi a relatada no artigo intitulado "O Comitê Cidadão como estratégia cogestiva em uma pesquisa participativa no campo da saúde mental” (Passos et al., 2013). 
Com uma média de 15 participantes em cada reunião, a composição geral do CAP contou com a participação de pesquisadores(as), trabalhadores(as), gestores(as) municipais e sujeitos da pesquisa, mas, em alguns municípios, participaram também outros atores, como familiares, coordenadores(as) dos Centros de Atenção Psicossocial (Caps), outros moradores(as) de Serviços de Residências Terapêuticas, movimento social, conselho de saúde e atores intersetoriais, como o ministério público. É interessante observar as reflexões sobre o componente "participação" dos diversos e diferentes atores do contexto das políticas de saúde, conforme apontado nas experiências de pesquisa descritas por Batista et al. (2010).

Na categoria de conteúdos discutidos repercussão/ recomendações e/ou encaminhamentos para o programa no âmbito municipal, perceberam-se a articulação necessária com Raps, a importância do dinheiro como ferramenta de desenvolvimento de habilidades múltiplas e a complexidade da vida cotidiana na qual os(as) beneficiários(as) estão inseridos, tendo sido algumas dessas conclusões traduzidas em encaminhamentos realizados a partir do CAP para os(as) gestores(as) e trabalhadores(as) dos municípios. Os processos de pesquisa participativa envolvem diferentes atores, entre os quais a gestão, que também é apontada no texto intitulado "Metodologia para análise da implantação de processos relacionados à descentralização da atenção à saúde no Brasil” (Vieira-da-Silva et al., 2005).

Os entraves na articulação das redes de cuidado em saúde mental, bem como o funcionamento dos próprios dispositivos que integram a Raps, também puderam ser discutidos em reuniões do CAP, já que interferiram de alguma maneira na realização da pesquisa. Lacunas nas informações registradas nos serviços sobre os(as) beneficiários(as), dificuldade de localização do(a) Técnico(a) de Referência de um(a) beneficiário(a) em algum serviço, fragilidades na dispensação de medicamentos, entre outros temas, puderam ser discutidos sem desviar do foco da pesquisa e do funcionamento do CAP. Pudemos observar aspectos semelhantes em pesquisas que exploram elementos que compõem a Raps com um olhar especial e valorizam, no processo, a participação dos seus integrantes (Vasconcelos, 2013).
Uma questão extremamente importante refere-se ao reconhecimento dos efeitos positivos da presença dos(as) pesquisadores(as) aos(às) beneficiários(as) no acompanhamento e na escuta diferenciada que eles(as) puderam fazer. Tais efeitos foram perceptíveis e narrados no CAP não só na relação entre beneficiários(as) e pesquisadores(as), mas também nas relações que cuidadores(as) e outros(as) trabalhadores(as) da Raps estabelecem com eles(elas).

Já na categoria processo da pesquisa em curso, uma pergunta frequente nas reuniões do CAP disse respeito aos achados parciais da pesquisa nacional. Havia curiosidade sobre se os temas locais também eram pautados em outros lugares, se os temas conflituosos eram semelhantes e quais as soluções encontradas nesses outros locais. Nesse sentido, a presença de técnicos(as) da Fiocruz nas reuniões locais foi fundamental para responder algumas perguntas e pensarmos juntos em algumas soluções, já que eles(as) acompanhavam nacionalmente a pesquisa.

Em acordo com Furtado e Nakamura (2014), o CAP não só facilitou o acesso aos sujeitos da pesquisa, como também possibilitou fazer uma devolutiva dos resultados nas suas particularidades em tempo real em cada município, ao passo que a participação dos diferentes sujeitos envolvidos se caracterizou pela horizontalidade e possibilidade de debate e encaminhamentos a cada pauta.

A experiência de Onocko-Campos et al. (2008) com o Comitê Científico apontou que a troca legítima entre profissionais e pesquisadores em um espaço democrático e criativo possibilitou maior articulação política e afetiva entre os atores, favorecendo a criação de uma rede entre serviços e as pessoas que os atravessam. Corroborando a experiência da autora, os CAP contaram com a participação de beneficiários(as) do programa, colocando o protagonismo deles(as) em ênfase ao garantir espaços de fala e voz a essas pessoas, centrais na política em questão, oportunizando o encontro, articulação e troca de afetos e opiniões.

Nesse sentido, o Comitê de Acompanhamento da Pesquisa pode ser considerado um microdispositivo (Barros, 1996; Lourau, 1993) na medida em que atua como espaço de trocas e construções coletivas 
em torno de problematizações que emergem no encontro, provocando deslocamentos do habitual. O CAP pode ser, então, entendido como dispositivo ativo, com capacidade de intervenção em realidades aparentemente fixas e estáveis e, consequentemente, de criação do novo - novos desejos, novas sensibilidades, novas configurações sociais e, portanto, novos arranjos quiçá mais inclusivos e criativos para a vida em sociedade. Segundo Barros (1996, p. 104), os dispositivos devem ser entendidos diante da sua

capacidade de irrupção naquilo que se encontra bloqueado de criar, no seu teor de liberdade em se desfazer dos códigos que procuram explicar dando a tudo o mesmo sentido, como o que tensiona, movimenta, desloca para outro lugar, provoca outros agenciamentos; como feito de conexões que ao mesmo tempo produzem outras conexões.

Dentre os efeitos do CAP como dispositivo, também pode ser destacada a abertura e ampliação para outros modos de fazer pesquisa, o que gera desconforto por reposicionar os "lugares" da universidade, dos serviços, da gestão, do(a) pesquisador(a) e dos sujeitos da pesquisa.

Enquanto potência criativa, produziu conexões novas e dinâmicas e provocou em quem pesquisa um sentimento de pertencimento ao processo que não poderia ser obtido em pesquisas com formatos engessados. Um exemplo foi a fala de um pesquisador, estudante de graduação em psicologia de uma das universidades envolvidas, quando disse que a pesquisa deu rumo às escolhas que precisava fazer na sua formação, permitindo construir um sentido para o curso e sua atuação futura, como pesquisador ou trabalhador da saúde mental. Outro exemplo: depois de uma discussão no CAP protagonizada por beneficiários incomodados com o controle dos trabalhadores sobre suas decisões, logo um consenso entre eles foi estabelecido.

\section{Considerações finais}

Em síntese, o CAP, com diferentes configurações, funcionou como um dispositivo "três em um": simultaneamente, dispositivo de interlocução, estabelecendo comunicação entre os atores sociais implicados(as) no processo de pesquisa, por meio do qual circulam opiniões, dúvidas e críticas; dispositivo de qualificação da pesquisa, na medida em que a definição de procedimentos e a análise das categorias passariam por processos de negociação entre diferentes atores; e dispositivo gerencial e de participação social, na medida em que permitiu, muitas vezes, sugerir recomendações e/ou encaminhamentos para a Raps no âmbito municipal.

O CAP é uma possibilidade interessante a ser considerada no caso de pesquisas avaliativas com desenho participativo que pretendam garantir espaços de debate sobre o andamento da pesquisa, além de um olhar coletivo e colaborativo sobre o fenômeno estudado. Ele pode assumir diferentes configurações, conforme organização, participação dos atores envolvidos e finalidade pactuada com o grupo.

Sugere-se que o CAP, para sua ampla potência, esteja inserido nos contextos de pesquisas participativas. Contudo, em outros desenhos de pesquisa, ele também pode contribuir para a qualificação de seu desenvolvimento, desde que seu formato considere o contexto e esteja alinhado ao objetivo da pesquisa.

\section{Referências}

BARROS, R. B. Dispositivos em ação: o grupo. Cadernos de Subjetividade, São Paulo, p. 97-106, jun. 1996. Número especial.

BATISTA, A. A. et al. A contribuição da pesquisa avaliação para o processo de implementação do controle social no SUS. Saúde e Sociedade, São Paulo, v. 19, n. 4, p. 784-793, 2010. Disponível em: <https://bit.ly/2XGwCBE>. Acesso em: 27 maio 2019.

DENZIN, N. K.; LINCOLN, Y. S. (Org.). $O$ planejamento da pesquisa qualitativa: teorias e abordagens. 2. ed. Porto Alegre: Artmed, 2006.

DIAZ, A. R. G. Pesquisa avaliativa em saúde mental: a regulação da "porta" nos Centros de Atenção Psicossocial. 2009. Dissertação (Mestrado em Saúde Coletiva) - Universidade Estadual de Campinas, Campinas, 2009. Disponível em: <https://bit.ly/2Wxmslt>. Acesso em: 8 maio 2019. 
FLICK, U. An introduction to qualitative research. London: Sage, 1998.

FURTADO, J. P.; NAKAMURA, E. (Org.). Inserção social e habitação de pessoas com sofrimento mental grave. São Paulo: Fap-Unifesp, 2014.

KOIZUMI, M. Fundamentos metodológicos da pesquisa em Enfermagem. Revista da Escola de Enfermagem da USP, v. 26, p. 33-47, 1992. Número especial.

LOURAU, R. Análise institucional e práticas de pesquisa. Rio de Janeiro: UERJ, 1993.

LYRA, J.; MEDRADO, B. Triangulação de pesquisadores como estratégia metodológica de pesquisa em saúde. In: BURITY, J.; RODRIGUES, C. M. L.; SECUNDINO, M. A. (Org.). Desigualdade e justiça social: diferenças culturais e políticas de identidade. Belo Horizonte: Argvmentvm, 2010.

v. 2. p. 35-45.

MATUS C. O plano como aposta. In: GIACOMONI, J.; PAGNUSSAT, J. L. Planejamento e orçamento governamental. Brasília: Enap, 2006. v. 1. p. 115-144.

MINAYO, M. C. S.; ASSIS, S. G.; SOUZA, E. R. (Org.). Avaliação por triangulação de métodos: abordagens de programas sociais. Rio de Janeiro: Editora Fiocruz, 2005.

ONOCKO-CAMPOS, R. T. et al. Pesquisa avaliativa em saúde mental: desenho participativo e efeitos da narratividade. São Paulo: Aderaldo \& Rothschild, 2008.

PASSOS, E. et al. O Comitê Cidadão como estratégia cogestiva em uma pesquisa participativa no campo da saúde mental. Ciência \& Saúde Coletiva, Rio de Janeiro, v. 18, n. 10, p. 2919-2928, 2013. Disponível em: <https://bit. ly/2XDQxkH>. Acesso em: 27 maio 2019.

SAUPE, R.; WENDHAUSEN, Á. L. P. Modelo matricial para construção de conhecimento no mestrado profissional em saúde. Revista Brasileira de Pós-Graduação, Brasília, DF, v. 3, n. 5, p. 107-116, 2006. Disponível em: <https://bit.ly/2LCfYAN>.

Acesso em: 28 jun. 2019.

SPINK, M. J. P. A ética na pesquisa social: da perspectiva prescritiva à interanimação dialógica. Revista Semestral da Faculdade de Psicologia da PUCRS, Porto Alegre, v. 31, n. 1, p. 7-22, 2000.

SPINK, M. J. P.; MENEGON, V. A pesquisa como prática discursiva. In: SPINK, M. J. P. (Org.).

Práticas discursivas e produção de sentidos no cotidiano: aproximações teóricas e metodológicas. São Paulo: Cortez, 1999. p. 63-92.

STAKE, R. E. A natureza da avaliação educacional. In: SANDER, J. (Org.). Introdução à avaliação de programas sociais. São Paulo: Instituto Fonte, 2003.

VASCONCELOS, E. M. Empoderamento de usuários e familiares em saúde mental e em pesquisa avaliativa/interventiva: uma breve comparação entre a tradição anglo-saxônica e a experiência brasileira. Ciência \& Saúde Coletiva, Rio de Janeiro, v. 18, n. 10, p. 2825-2835, 2013. Disponível em: <https://bit.ly/2KIXInO>. Acesso em: 11 jun. 2019.

VIEIRA-DA-SILVA, L. M. et al. Metodologia para análise da implantação de processos relacionados à descentralização da atenção à saúde no Brasil. In: HARTZ, Z. M. A.; VIEIRA-DA-SILVA, L. M. (Org). Avaliação em saúde: dos modelos teóricos à prática na avaliação de programas e sistemas de saúde. Rio de Janeiro: Fiocruz, 2005. p. 207-253.

ZUÑIGA, R.; LAPERRIÈRE, H. Avaliação comunitária: conflitos verticais e ambiguidades metodológicas. In: BOSI, M. L. M.; MERCADO, F. J. (Ed.). Avaliação qualitativa de programas de saúde: enfoques emergentes. Petrópolis: Vozes, 2006. p. 118-144.

\section{Contribuição dos autores}

Todos os autores conceberam e redigiram igualmente o artigo.

Recebido: 27/05/2019

Aprovado: 03/06/2019 


\section{ERRATA}

Na apresentação "Entre desafios e aberturas possíveis: vida em liberdade no contexto da desinstitucionalização brasileira”, doi 10.1590/So104-12902019000003, publicado no volume 28, número 3, 2019, a seção está incorreta. Onde se lia:

ARTIGOS

Leia-se:

DOSSIE

No artigo “O Programa de Volta para Casa na vida cotidiana dos seus beneficiários”, doi 10.1590/So10412902019190435, publicado no volume 28, número 3, 2019, a seção está incorreta.

Onde se lia:

ARTIGOS

Leia-se:

DOSSIÊ

No artigo "Produção compartilhada de conhecimentos em saúde mental: o Comitê de Acompanhamento de Pesquisa", doi 10.1590/So104-129020190436, publicado no volume 28, número 3, 2019, a seção está incorreta. Onde se lia:

ARTIGOS

Leia-se:

DOSSIÊ

No artigo "Construindo histórias em tessitura lenta: desinstitucionalização e narrativas em pesquisa", doi 10.1590/So104-129020190428, publicado no volume 28, número 3, 2019, a seção está incorreta.

Onde se lia:

ARTIGOS

Leia-se:

DOSSIÊ

No artigo "Narrativas e sentidos do Programa de Volta para Casa: voltamos, e daí?", doi 10.159o/So10412902019190429, publicado no volume 28, número 3, 2019, a seção está incorreta.

Onde se lia:

ARTIGOS

Leia-se:

DOSSIÊ

No artigo "A rede embala e o ritmo da gestão embola", doi 10.1590/So104-12902019190443, publicado no volume 28, número 3, 2019, a seção está incorreta.

Onde se lia:

ARTIGOS

Leia-se:

DOSSIÊ 